\title{
Yeast membrane proteomics using leucine metabolic labelling: bioinformatic data processing and exemplary application to the ER-intramembrane protease Ypf1
}

Lars Nilse ${ }^{1,7}$, Dönem Avci' ${ }^{2,6,7}$ Patrick Heisterkamp ${ }^{1}$, Oliver Serang ${ }^{3,4}$, Marius K. Lemberg ${ }^{2, \star}$, Oliver Schilling ${ }^{1,5, *}$

1. Institute of Molecular Medicine and Cell Research, University of Freiburg, D-79104 Freiburg, Germany

2. Zentrum für Molekulare Biologie der Universität Heidelberg (ZMBH), DKFZ-ZMBH Allianz, Im Neuenheimer Feld 282, 69120 Heidelberg, Germany

3. Freie Universität Berlin, Department of Informatik, Takustr. 9, 14195 Berlin, Germany

4. Leibniz-Institute of Freshwater Ecology and Inland Fisheries (IGB), Müggelsee 310, 12587 Berlin, Germany

5. BIOSS Centre for Biological Signalling Studies, University of Freiburg, D-79104 Freiburg, Germany

6. Present address: Ludwig Institute for Cancer Research, University of Oxford, Oxford, OX3 7DQ, UK

7. equal contribution

*Correspondence: Email: m.lemberg@zmbh.uni-heidelberg.de; oliver.schilling@molmed.uni-freiburg.de

Running Title: Leucine metabolic labelling 


\section{Abbreviations}

d3-leucine, trideuterated leucine

ER, endoplasmic reticulum

ERAD, endoplasmic reticulum associated degradation

LC-MS, liquid chromatography - mass spectrometry

PSM, peptide-to-spectrum matches

SILAC, stable isotope labeling by amino acids in cell culture 


\section{Summary}

We describe in detail the usage of leucine metabolic labelling in yeast in order to monitor quantitative proteome alterations, e.g. upon removal of a protease. Since laboratory yeast strains are typically leucine auxotroph, metabolic labelling with trideuterated leucine (d3-leucine) is a straightforward, cost-effective, and ubiquitously applicable strategy for quantitative proteomic studies, similar to the widely used arginine/lysine metabolic labelling method for mammalian cells. We showcase the usage of advanced peptide quantification using the FeatureFinderMultiplex algorithm (part of the OpenMS software package) for robust and reliable quantification. Furthermore, we present an OpenMS bioinformatics data analysis workflow that combines accurate quantification with high proteome coverage. In order to enable visualization, peptide-mapping, and sharing of quantitative proteomic data, especially for membrane-spanning and cell-surface proteins, we further developed the webapplication Proteator (http://proteator.appspot.com). Due to its simplicity and robustness, we expect metabolic leucine labelling in yeast to be of great interest to the research community. As an exemplary application, we show the identification of the copper transporter Ctr1 as a putative substrate of the ER-intramembrane protease Ypf1 by yeast membrane proteomics using d3-leucine isotopic labeling. 


\section{Introduction}

A long-standing problem in protease research has been the identification of substrates [1]. There exist robust methods for the identification of cleavage products [2]. However, they are not suited for metastable cleavage fragments that are rapidly degraded. One class of proteases that trigger rapid turnover of cleavage fragments are intramembrane proteases, which are unusual proteases that cleave their substrates commonly within transmembrane anchors thereby releasing peptide fragments from the membrane [3]. Like for classical proteases, this intramembrane cut generates neo-Nand C-termini. When released into the cytoplasm, these new termini are recognized by cellular quality control systems such as the $\mathrm{N}$-end rule pathway and the ubiquitin proteasome system [3]. Likewise, the mitochondrial rhomboid intramembrane protease PARL generates a so-called $\mathrm{N}$-degron in the PINK1 cleavage product that is recognized by the E3 ubiquitin ligase Ubr1, which in turn flags it for proteasomal degradation [4]. A direct coupling between intramembrane proteases and proteasomal degradation is observed in the endoplasmic reticulum (ER) [5].

The ER-associated degradation (ERAD) pathway serves as an important cellular safeguard by directing incorrectly folded and unassembled proteins from the ER to the proteasome [6]. Work by a number of laboratories has revealed several factors that mediate recognition and targeting of a heterogeneous substrate spectrum, forming several parallel degradation routes. In this regard, budding yeast Saccharomyces cerevisiae helped to break down this complexity and to work out the basic principle of ERAD. Translocation across the ER membrane is assumed to depend on a proteinconducting channel, but the identity of this so-called 'dislocon' is still controversial [6]. The commonly accepted view is that Cdc48 (known as p97 or VCP in mammalian cells) extracts misfolded proteins from the ER membrane as unfolded, full-length polypeptides. However, Cdc48/p97-independent release of aberrant proteins from the ER has been observed [7] and exceptions to the ERAD dislocation mechanism exist [6]. One alternative mechanism that releases TM segments from cellular membranes involves intramembrane proteolysis, which introduces an irreversible step in protein dislocation [5]. For example, the yeast aspartate protease Ypf1, a homologue of the signal peptide peptidase, functionally interacts with the E3 ligase Doa10 and the rhomboid pseudoprotease Dfm1 to regulate the abundance of plasma membrane nutrient transporters [8]. We previously showed that the high-affinity zinc transporter Zrt1 is degraded in a zinc dependent manner by Ypf1, showing that cleavage by an intramembrane protease executes fast and tight abundance control of this physiologically important membrane protein [8].

Global quantitative proteomic profiling has enabled protease substrate discovery in numerous cases [9-11]. Likewise, mass-spectrometry based proteomics approaches have also enabled the identification of endogenous substrates for the ERAD machinery [12]. Recently, we have used quantitative proteomic profiling of yeast microsomes to identify novel Ypf1 substrates, which accumulate upon genetic deletion of this intramembrane protease [8]. This work led to the identification of the zinc transporter Zrt1 as a novel Ypf1 substrate. 
Quantitative proteome profiling is often based on the metabolic incorporation of stable isotope labelled amino acids. In mammalian cells, lysine and arginine are most widely used for this purpose, with the highly popular "stable isotope labeling by amino acids in cell culture" (SILAC) [13] approach being almost an acronym for quantitative proteomics. However, even before the advent of SILAC, proteomic studies in yeast have employed d3-leucine for metabolic labeling [14]. Since commonly used yeast strains are auxotroph for leucine, usage of leucine for metabolic labeling is a straightforward and cost-effective approach to enable quantitative proteomic studies in yeast. This is especially true for multi-step sample preparation techniques (such as microsome preparation) for which metabolic labeling is preferred, since it enables samples mixing at an early stage, thus minimizing experimental bias [15]. Indeed, our previous finding of Zrt1 being a Ypf1 substrate is based on leucine metabolic labelling [8].

In this report, we present a robust bioinformatics workflow for the analysis of quantitative proteomic data based on the incorporation of d3-leucine. We highlight precise peptide feature detection and quantification using advanced tools of the OpenMS pipeline [16]. Based on this analysis, we show that the copper transporter Ctr1 is a Ypf1 substrate.

\section{Materials and Methods}

Yeast strains and plasmids

Yeast strains used in this study are derived from W303 background and listed below. Genetic manipulations were performed using a standard PEG/LiAC protocol [17]. Genomic tagging of proteins and individual gene deletions were performed by standard PCR-based homologous recombination $[18,19]$.

\begin{tabular}{|l|l|l|}
\hline Strain & Description & Source \\
\hline W303 & MATa \{ade2-1 can1-100 his3-11,15 leu2-3,112 trp1-1 ura3-1 & \\
\hline YDA11 & MATa $\triangle$ ypf1::HIS3 & {$[8]$} \\
\hline YDA61 & MATa CTR1::pYM22-3HA-kITRP1 & This study \\
\hline YDA62 & MATa $\triangle$ ypf1::HIS3, CTR1::pYM22-3HA-kITRP1 & This study \\
\hline YDA251 & MATa sec23-1, pRS316-prGAL1-3HA-Ctr1 & This study \\
\hline YDA252 & MATa sec23-1 $\triangle$ ypf1::HIS3, pRS316-prGAL1-3HA-Ctr1 & This study \\
\hline Plasmid & & \\
\hline PDA62 & pRS316-prGAL1-3HA-Ctr1 & This study \\
\hline
\end{tabular}

\section{Antibodies}

The following antibodies were used: mouse monoclonal anti-HA (HA.11; Covance), rabbit polyclonal anti Sec61 and rabbit polyclonal anti Emp47 were obtained from B. Dobberstein.

\section{Cellular fractionation}

The wt and $\Delta y p f 1$ yeast strains were grown at $30^{\circ} \mathrm{C}$ in synthetic complete 
medium $(0.67 \%$ yeast nitrogen base with ammonium sulfate-without amino acids and $2 \%$ glucose, containing the appropriate supplements for the strains' auxotrophy) supplemented with $100 \mathrm{mg} / \mathrm{l}$ of d3-leucine (Euroisotopes) or the corresponding isotopically normal leucine. ER enriched microsomes were prepared as has been described previously [20], Fig. 1 . In brief, cells were grown to OD600 0.5-0.6, 250-300 OD of wild type and $\Delta y p f 1$ cells were mixed with 1:1 ratio prior to preparation and harvested. All following steps were performed at $4{ }^{\circ} \mathrm{C}$. Cell pellet was resuspended in 1/100 culture volume of low-salt buffer [20 mM HEPES-KOH pH 7.6, $50 \mathrm{mM}$ potassium acetate, $5 \mathrm{mM}$ magnesium acetate, $1 \mathrm{mM}$ EDTA, $1 \mathrm{mM}$ dithiothreitol (DTT, Sigma), protease inhibitors; $1 \mathrm{mM}$ phenylmethane sulfonyl fluoride (Applichem), $10 \mu \mathrm{M}$ E-64 (Sigma), $10 \mathrm{mM}$ o-phenanthroline (Applichem), $1 \mu \mathrm{M}$ Pepstatin-A (Sigma)]. Two cell volumes of glass beads were added and cells were lysed by vortexing 5 times for $1 \mathrm{~min}$, with 1-min intervals on ice. Crude lysate was obtained by centrifugation at $1200 \times \mathrm{g}$ for 2 min. ER-enriched membrane fraction was obtained by centrifugation at $6000 \times \mathrm{g}$ for 20 $\min$.

ER-enriched microsome pellet was resuspended in low-salt buffer and 400 $\mathrm{mM}$ potassium acetate and $50 \mathrm{mM}$ EDTA were added. After 15 min incubation on ice, membranes were collected by centrifugation at $140000 \times \mathrm{g}$ for $1 \mathrm{~h}$ on $0.5 \mathrm{M}$ sucrose cushion. High salt/EDTA washed membrane pellet was resuspended in low-salt buffer. In order to enrich for integral membrane proteins, 1 volume of freshly prepared ice-cold $100 \mathrm{mM}$ sodium carbonate solution was added and incubated for $15 \mathrm{~min}$ on ice. Sample was loaded on alkaline sucrose cushion $(100 \mathrm{mM}$ sodium carbonate and $250 \mathrm{mM}$ sucrose) and membranes were collected by centrifugation at $130000 \times \mathrm{g}$ for $10 \mathrm{~min}$. Membrane pellet was frozen in liquid nitrogen and kept at $-80^{\circ} \mathrm{C}$.

\section{Sample Preparation and Mass Spectrometric Analysis}

The microsome suspension was precipitated by acetone-methanol in order to remove lipids. The protein pellet was re-solubilized in ice-cold $100 \mathrm{mM} \mathrm{NaOH}$ using ultrasonication. After adjusting the $\mathrm{pH}$ to 8.0 using HEPES, the samples were trypsinized (1:100; sequencing grade trypsin, Worthington; for $16 \mathrm{~h}$ at $37^{\circ} \mathrm{C}$ ), reduced (2 mM tris(2-carboxyethyl)phosphine (TCEP; Sigma), one hour at $37^{\circ} \mathrm{C}$ ), and alkylated (10 mM iodoacetamide (Sigma) at $37^{\circ} \mathrm{C}$ for one hour followed by $5 \mathrm{mM}$ dithiothreitol (DTT; Sigma), 20 min at $22^{\circ} \mathrm{C}$ for quenching). The peptide sample was desalted using C18 solid phase extraction (Waters) according to manufacturer's instruction and fractionated by strong cation exchange (SCX) chromatography as described previously [21]. Following SCX elution, fractions were desalted by STAGE tips [22] prior to liquid chromatography - tandem mass spectrometry (LC-MS/MS) analysis, which was performed using an Orbitrap $\mathrm{XL}$ as described previously $[8,23]$. The mass spectrometry proteomics data have been deposited to the ProteomeXchange Consortium [24] via the PRIDE partner repository with the dataset identifier PXD003052.

\section{MS and bioinformatics data analysis}

d3 leucine metabolic labelling [14] is a less common labelling technique that may require specific adaptions in some software packages. Here we employed the OpenMS software framework, which allows for the design of highly modular data analysis workflows that can easily be adapted to non-standard experimental techniques 
such as d3 leucine labelling. The TOPPAS OpenMS workflow can be downloaded at http://www.mol-med.uni-

freiburg.de/mom/schilling/protein_quantification_Leu3_LTQOrbiXL_20160406.

Raw files were converted to mzML [25] using ProteoWizard's msconvert [26]. Peptide-to-sequence matching was performed using MS-GF+ [27] with a Saccharomyces cerevisiae Uniprot database downloaded in July 2015, counting 6721 entries and appended with an equal number of randomized sequences. Search parameters included: high resolution mass spectrometer settings, tryptic cleavage specificity, iodoacetamide protection of cysteine, and variable d3-leucine modification.

Peptides were probabilistically assembled into proteins using Fido [28,29]. Peptides were quantified using FeatureFinderMultiplex [30]. Peptide mapping was performed using Proteator (http://proteator.appspot.com), a web-based application which encompasses the functionality of the previously published peptide mapping tool Improviser [31]. Proteator maps each peptide to its respective location on the protein and uses Uniprot [32] data for protein sequence and topology.

\section{Chase experiments}

Promoter shut-off and cycloheximide chase experiments were performed with cells growing in exponential phase. For promoter shut-off chase experiments cells were grown at $25^{\circ} \mathrm{C}$ in rich medium (1\% Bacto-yeast extract, 2\% Bacto-peptone) with 2\% raffinose. Protein expression was induced for $1 \mathrm{~h}$ at $33^{\circ} \mathrm{C}$ in rich medium with $2 \%$ galactose followed by a promoter shut-off/chase at $33^{\circ} \mathrm{C}$ in rich medium with $2 \%$ glucose. For cycloheximide chase experiments shown cells were grown at $30^{\circ} \mathrm{C}$ in rich medium with $2 \%$ glucose. Cycloheximide was added with $100 \mu \mathrm{g} / \mathrm{ml}$ final concentration. For all chase analyses, $1 \mathrm{ml}$ samples were taken at indicated time points and total cell extracts were prepared by alkaline lysis followed by precipitation with trichloracetic acid. Samples were then analyzed by western blotting with the corresponding antibody. Sec61 western blots were performed as a loading control. Results were reproduced with two isogenic clones for each strain.

\section{Western blotting}

Total cell extracts and subcellular factions were solubilized in SDS sample buffer (50 mM Tris-Cl pH 6.8, 10 mM EDTA, 5\% glycerol, 2\% SDS, 0.01\% bromphenol blue) containing $5 \% \beta$-mercaptoethanol. All samples were incubated for $15 \mathrm{~min}$ at $65^{\circ} \mathrm{C}$. For western analysis, proteins were separated by SDS-PAGE using Tris-glycine acrylamide gels and transferred to PVDF membrane followed by enhanced chemiluminescence analysis using X-ray films or the LAS-4000 system (Fuji). Data shown are representative of three independent experiments. For quantification, we used the Multi Gauge software (Fuji) and data acquired from the LAS-4000.

\section{Results}

Overview 
As described previously [8], we employed a quantitative proteomic strategy to identify novel substrates of the yeast intramembrane protease Ypf1. We hypothesized that loss of Ypf1 results in the accumulation of its membrane-spanning substrates. We employed d3-leucine metabolic labelling for quantitative comparison of the microsomeenriched sub-proteome. Metabolic labelling enables mixing of different samples prior to sample fractionation (Fig 1), thus increasing sample comparability. We probed for the enrichment of ER-residual proteins by immunoblotting analysis of the ER-marker Sec61 and Emp47 (Fig 1), a membrane protein localized to ER-derived vesicles, which is posttranslationally modified in the secretory pathway. The ER resident form of Emp47 is differentiated from the modified forms by its lower molecular weight.

\section{OpenMS workflow for LC-MS/MS data analysis}

We established an OpenMS workflow for the in-depth analysis of d3-leucine LC-MS/MS data. OpenMS is a modular environment for the analysis LC-MS/MS data, enabling the design of integrated analysis workflows even for non-standard experimental techniques such as d3-leucine labelling. The workflow consists of fourteen different tools and can be divided into four major parts (Fig. 2a) with numerous individual processing steps (Fig. 2a / b). It is important to notice that peptide sequence information ("peptide ID") and quantitative peptide information ("peptide quant.", also referred to as peptide features") are determined independently of each other. Sequences are mapped to peptide features at a subsequent step ("ID mapping"). The complete set of proteins is then inferred from the peptide sequences ("protein inference"). In a final step, the protein information and the quantitative peptide information are combined, and a fold change for proteins calculated.

\section{Proteome coverage}

The combination of the MS-GF+ search engine and Fido for protein inference resulted in 2,850 non-redundant protein identifications at a false-discovery rate (FDR) of $1 \%$. Note that neither the search engine MS-GF+ nor the inference algorithm Fido are part of the OpenMS project. However, OpenMS provides adapters for the easy integration of both external tools into the workflow. We have previously analysed this dataset using X!Tandem [33], PeptideProphet [34], and ProteinProphet [35] as part of the Trans-Proteomic Pipeline (TPP) [36]. The TPP-based approach yielded 2,682 protein identifications at a $1 \%$ FDR. Our re-analysis compares favourably to the earlier analysis. In both cases, only a fraction of the identified proteins was amenable to quantification, since some peptides lack a leucine residue (Fig 3a).

Fido computes posterior probabilities for each protein (i.e., the chances that each protein is in the sample). Because it is probabilistic, it has been shown to outperform non-probabilistic methods such as parsimony, which effectively threshold conclusions regarding protein presence into either a 0 or a 1 rather than respecting the inherent uncertainty in protein inference [37]. Unlike other probabilistically motivated methods for protein inference, Fido uses a generative probability model of how each possible set of proteins would emit every possible set of peptides. This generative model enables shared peptides to be accounted for properly with all possible outcomes: a peptide shared between two proteins could be created by neither protein, exactly one protein, or both proteins. Other probabilistically motivated methods like ProteinProphet 
[35] achieve computational efficiency by directly running inference backwards, i.e. inferring peptides from spectra and then inferring proteins from peptides. Because no generative model is used by such methods, it is ambiguous as to which protein (or set of proteins) created any given shared peptide. ProteinProphet attempts to resolve this problem by heuristically iterating and partitioning the ownership of a shared peptide as proportional to the confidence that each protein is present; however, this iterative method has been demonstrated to be unstable for very large data sets, and can substantially overestimate or underestimate protein probabilities $[28,38]$.

Although the generative model resolves shared peptides in a desirable manner, it does so at the cost of introducing a significant computational burden. MSBayes, another high-quality Bayesian tool for protein inference, approaches this computational burden by using Markov chain Monte Carlo (MCMC) to estimate protein posterior probabilities [39,40]. Fido approaches this graphical Bayesian inference problem by effectively enumerating every possible set of present proteins; however, where naive enumeration would require a possible exponential number of steps to perform, Fido exploits properties of the model and the graph to perform inference very efficiently, and automatically balances the discrimination (i.e., awarding higher probabilities to non-decoy and present proteins) and calibration (i.e. ensuring that a protein awarded a $70 \%$ probability has only a $30 \%$ chance of being identified in error), and thereby control the false discovery rate (FDR).

Peptide data were processed with Fido using a default protein clique size of 17. The proteins with a global protein-level FDR less than $1.0 \%$ were used for further processing.

\section{Quantification}

The tool "FeatureFinderMultiplex" detects peptide feature pairs and computes their ratios. We have previously shown that the underlying algorithm accurately detects and quantifies peptide features even at comparably low signal-to-noise ratios [30]. One test dataset consisted of trypsin-digested Escherichia coli lysate, isotopically labelled by reductive dimethylation using $\mathrm{H}_{2} \mathrm{CO}$ or $\mathrm{D}_{2} \mathrm{C}^{13} \mathrm{O}$, and mixed at a heavy:light ratio of $4: 1$ $\left(\log _{2}\right.$ value $\left.=2.0\right)$. On the protein level, the quantitation algorithm displayed a median, $\log _{2}$-transformed heavy:light ratio of 1.78 with a standard deviation of 0.58 . This outperformed the widely used XPRESS algorithm [41] which displayed a standard deviation of 1.50 [30]. A second test data set consisted of human embryonic kidney cell lysates, which were trypsin-digested, isotopically labelled by reductive dimethylation using $\mathrm{H}^{2} \mathrm{CO}$ or $\mathrm{D}_{2} \mathrm{C}^{13} \mathrm{O}$, and mixed at a heavy:light ratio of 1:4 $\left(\log _{2}\right.$ value $\left.=-2.0\right)$ [42] On the protein level, the quantitation algorithm displayed a median, $\log _{2}$-transformed heavy:light ratio of -1.91 with a standard deviation of 0.56 .

The OpenMS-based re-analysis yielded 2,126 protein quantifications with a standard deviation of 0.45 , slightly outperforming the TPP-based 2,095 quantifiable proteins with a standard deviation of 0.82 . 1,724 proteins were quantified in both analyses. Most proteins display a fold-change (fc-) value (log 2 light/heavy) close to zero with a near-symmetrical distribution of positive and negative Fc-values (Fig $\mathbf{3 b}$ ). Noteworthy, the re-analysis corroborates the initially observed accumulation of the zinc transporter Zrt1 upon Ypf1 deletion (Sup. Figure 1) [8]. 
Robust, yet accurate detection and quantification of peptide features in LC-MS data is key to any quantitative proteomic analysis, which is based on MS1 data. We have previously shown that integration of data in both RT and $\mathrm{m} / \mathrm{z}$ dimension improves quantification accuracy, especially for low and medium abundance peptides that are associated with LC-MS data of limited signal-to-noise ratios [30]. In our workflow, peptide quantification starts with a noise reduction step using a moving average in RT direction, followed by the detection of peptide feature pairs using the FeatureFinderMultiplex tool (Fig 2).

The FeatureFinderMultiplex algorithm requires several settings for feature detection. First, the possible charge states need to be specified (charge $=1+$ to $7+$ ). We then characterize the liquid chromatography by specifying a typical and a minimal feature elution time (rt_typical $=70 \mathrm{~s}$, rt_min $=5 \mathrm{~s}$ ). The mass-to-charge tolerance sets a limit on the possible mass shift error between isotopic peaks ( $\mathrm{mz}$-tolerance $=5 \mathrm{ppm}$ ). The signal intensity cutoff we set very low in order to detect most of the low intensity peptides (intensity_cutoff $=10$ ). Finally, we compare the isotopic pattern to a theoretical averagine distribution (averagine_similarity $=0.9$ ) and the two isotopic patterns within a peptide feature pair (peptide_similarity $=0.85$ ). A parameter close to one ensures that the isotopic pattern resembles a typical (average) peptide in this mass range and that isotopic patterns of both light and heavy peptide feature are identical. Note that the peptide features are not detected individually and then paired off, but detected directly. This approach is advantageous since feature pairs with a known fixed $\mathrm{m} / \mathrm{z}$ shift show clearer signatures in the spectral data than single features.

Peptide quantification based on $\mathrm{d} 3$ leucine labelling poses particular challenges. For example, peptides with a single leucine in their sequence exhibit a small mass shift of $3 \mathrm{Da}$, which may result in partial overlap with higher natural isotopes of the "light" counterpart. However, the feature detection tools employed in our analysis are capable of successfully distinguishing such overlapping features. This is illustrated in Fig $\mathbf{3 c}$ for the peptide YLISPDVHPAQNQNIQENK, which contains a single leucine.

The mass difference between light and heavy features is useful in corroborating sequence identifications. In the case of Leu D3 labelling, the mass difference of a co-eluting feature pair should match the number of leucine residues in the identified peptide. For example, a mass difference of $3 \mathrm{Da}$ is rightfully linked to a peptide sequence with a single leucine. In a further example (Fig. 3d) the two-leucine peptide TASEVPAHLTVYDIAGLTK is rightfully linked to a feature pair that differs by 6 $\mathrm{Da}$, i.e. $3 \mathrm{Th}$ at charge $2+$. The deuterium in the labelled peptide also results in a slightly earlier elution. This retention time shift does not impede peptide feature quantification. Such cross-validation may be useful when deciding on which proteins to focus in subsequent corroborative experiments (e.g. immuno-blotting). The complexity and combinatorial possibilities of metabolic and chemical labeling strategies have, to date, prevented a fully automated tackling of these issues. However, FeatureFinderMultiplex produces feature maps, which can be visualized by TOPPView [43] and overlaid with peptide identification maps as well as raw LC-MS data for manual inspection. We consider it useful to perform this step for selected individual peptides and proteins prior to follow-up experiments. 
After peptide feature quantification, the independently determined peptide sequence and peptide fold-change information are combined. For that, the FDR-filtered PSMs are mapped to the detected peptide pairs. Furthermore, we remove any peptide feature pairs to which a sequence without leucine was mapped, since they are clearly false positives: only peptides with at least one leucine experience a mass shift, and can therefore provide quantitative information. Note that peptide sequences without leucine are not entirely ignored in our analysis. They contribute, as do all other sequences, to protein inference. Finally, after merging the peptide information from all fractions, we provide the peptide fold changes and the complete list of proteins from the inference to the protein quantification algorithm. The algorithm determines the fold changes for as many proteins as possible. The entire workflow yields output in a simple csv text format. As mentioned above, our workflow quantified a total of 2,126 proteins.

\section{Peptide mapping with "Proteator"}

We have previously highlighted the usefulness of peptide mapping in combination with protein topographical annotation for the interpretation of quantitative proteomic data [31]. However, our previous tool was specifically designed for xml data of the TPP. For improved compatibility, we are now introducing Proteator (http://proteator.appspot.com), a web-based application hosted on a server provided by Google that maps each peptide to its respective location on the protein. Upon input (csv, tsv, mzTab files [44]) data concerning sequence and topology of the proteins is retrieved from Uniprot, the peptides are mapped to their positions and the result is displayed in the web browser. Proteator includes advanced analysis functionality such as visual display of fold changes or the ability to filter proteins for certain topological domains. The finished visualization can be exported as .html file for ease of sharing. Exemplary peptide mapping for the protein Ctr1 is shown in Fig 4a. The open source code is available at https://github.com/Proteator.

Ctr1 is a Ypf1 substrate candidate

As previously outlined, we reasoned that putative Ypf1 substrates accumulate upon loss of Ypf1. In our previous proteomic analysis, this rationale led to the identification of the zinc transporter Zrt1 as the first known polytopic transmembrane protein that is cleaved by Ypf1 [8].

In the present analysis, Ctr1 raised our interest for multiple reasons. Firstly, like the confirmed Ypf1 substrate Zrt1, also Ctr1 is a polytopic transmembrane protein. Secondly, four peptides consistently and collectively indicate an increase in Ctr1 abundance upon loss of Ypf1 (see also below). Thirdly, Ctr1 is a copper transporter and we have recently shown that the abundance and activity of the functionally related zinc transporter Zrt1 is controlled by Ypf1[8]. For each Ctr1 peptide, the heavy counterpart (representing $\Delta y p f 1$ ) yields a larger integrated signal than the corresponding light counterpart (representing wild-type yeast) (Fig 4b). The global heavy-to-light ratio for Ctr1 is 1.25 (log2 fold-change 0.33 ).

Our previous analysis using a high content screening approach based on the yeast GFP library [45] indicated that Ctr1-GFP accumulates in Ypf1 deficient strains suggesting like Zrt1 it is substrate of Ypf1-dependent ERAD [8]. Proteomics analysis 
now confirms that this effect is physiologically relevant and not caused by the spacious GFP tag. Consistent with this, a cell-based ERAD assay using an inducible HA-tagged Ctr 1 construct shows an extended half-life of Ctr1 in $\square$ ypfl cells compared to control cells (Fig. 5a). Likewise, chromosomally tagged Ctr1 shows an increased steady state level and a reduced turnover in Ypf1 deficient strains (Fig. 5b). Taken together, these results give compelling evidence that Ctr1 is a substrate of Ypf1. Furthermore, these results demonstrate that the workflow presented here allows efficient and robust analysis of d3-leucine metabolic labelling results.

\section{Discussion}

We present a modular approach for the analysis of d3 leucine metabolically labelled LC-MS/MS data and showcase the identification of the copper transporter Ctr1 as a candidate for the intramembrane protease Ypf1. Since Ypf1 also regulates the levels of the zinc transporter Zrt1 [8], there appears to be emerging evidence that intramembrane proteases contribute to cellular transition metal homeostasis by proteolysis of the corresponding transmembrane transport proteins.

Bioinformatically, we focused on the emerging OpenMS platform [16], which is characterized by an unparalleled degree of modularity. As such, it is a viable alternative to the more established TPP platform and offers workflow integration for an expanding set of novel proteomic data analysis tools. Arguably, most of these abilities may be predominantly of interest for rather advanced data analysis needs. However, it is our opinion that this situation justifies the development and usage of high-performance modular platforms for the investigation of LC-MS/MS data. The present workflows are easily adjustable to other MS1 labeling techniques. The algorithms and the OpenMS environment have already been used for the analysis of dimethyl-labelling [30,42]. Exemplary workflows for dimethylation-based quantitative proteomics can be downloaded from http://openms.de/workflows.

\section{Acknowledgements}

OS acknowledges support by the Deutsche Forschungsgemeinschaft $(\mathrm{SCHI}$ $871 / 2$ and SCHI 871/5, SCHI 871/6, GR 1748/6, INST 39/900-1) and SFB850-Project B8, European Research Council (ERC-2011- StG 282111-ProteaSys), and the Excellence Initiative of the German Federal and State Governments (EXC 294, BIOSS). MKL acknowledges support by the Baden-Württemberg Stiftung and the Deutsche Forschungsgemeinschaft (SFB 1036, TP 12).

\section{Figure Legends}

Figure 1: Experimental outline of the fractionation protocol. Wild-type and $\Delta y p f 1$ cells were compared at steady state conditions. d3-leucine was used for metabolic labeling. 
Cellular fractionation, high salt/EDTA wash and sodium carbonate extraction were performed to enrich membrane proteins and immunoblot analysis was used to characterize the membrane-enriched fractions, of which only the ER enriched membrane fraction was used for proteomic analysis. Sec61 is shown as an ER marker. Emp47 is a membrane protein localized to ER-derived vesicles and gets posttranslational modifications through the secretory pathway. ER resident form (indicated with arrow) could be differentiated from the modified form due to its different mobility on SDS-PAGE.

Figure 2a/b: OpenMS workflow for the analysis of d3-leucine labelled data. The workflow can be divided into five parts. Peptide sequence identification followed by protein inference. Peptide feature quantification followed by peptide sequence mapping and final protein quantification. The tools are referenced by their numbers, 3 to 20 , in the text. The final protein list can be exported to the standard file format mzTab, and then further explored with the Proteator viewer. (In the depicted workflow, peptide sequence identification is performed using a single search with variable modifications. In the analysis, two separate search runs with different fixed modifications were performed instead, and subsequently combined.)

\section{Figure 3:}

(a) Frequency of leucine in PSMs and quantified peptide feature pairs. The dark grey bars represent all identified PSMs and correspond to the output of tool 6 in Fig. 2a. The light grey bars are the subset of these PSMs, which could be mapped to peptide features. The red bars indicate quantified peptide features to which one or multiple PSMs can be mapped (output of tool 15 in Fig. 2a).

(b) Histogram of $\log _{2}$ light:heavy values

(c) D3-leucine peptide feature pair with 6 Da mass shift in LC-MS data. (left) labelled and unlabelled peptide features of the same sequence in the $\mathrm{m} / \mathrm{z}-\mathrm{RT}$ plane in 3D view (right) peptide pair in a single spectrum at RT $=3,852 \mathrm{~s}$

(d) Overlapping peptide feature pair with $3 \mathrm{Da}$ mass shift. (left) peptide features of the same sequence in the $\mathrm{m} / \mathrm{z}-\mathrm{RT}$ plane in $3 \mathrm{D}$ view (right) peptide pair in a single spectrum at $\mathrm{RT}=2,560 \mathrm{~s}$

The red arrows indicate the mass shift between the isotopes of a peptide ( 0.5 Th for charge $2+)$ as well as the mass shift for a single leucine label (1.5 Th for charge $2+$ ) or a double leucine label (3 Th for charge 2+).

\section{Figure 4:}

(a) Exemplary Proteator output

(b) Peptide evidence for the copper transporter Ctr1. Four different peptides were both identified and quantified, corresponding to a $13.8 \%$ sequence coverage. The transmembrane regions are highlighted (TM). Each of the four peptide-feature pairs is shown in the $\mathrm{m} / \mathrm{z}-\mathrm{RT}$ plane in 3D view. fc, fold-change (log2 of ratio heavy / light = $\Delta y p f 1$ / control) 


\section{Figure 5:}

(a) Promoter shut-off/chase experiments in the temperature sensitive sec23-1 background, which blocks COPII-dependent ER to Golgi trafficking at restrictive temperature of $33^{\circ} \mathrm{C}$, show a significant delay in the degradation of newly synthesized, ER-resident Ctr1 in the absence of Ypf1, compared to the control strain (means \pm SEM, $\mathrm{n}=3$ ). The unrelated ER protein Sec61 is used as a loading control.

(b) Cycloheximide ( $\mathrm{CHX}$ ) chase experiments with wt and $\Delta y p f 1$ cells expressing chromosomally tagged Ctr1 show increased steady state level (time point 0 ) and slower degradation kinetics upon ypf1 deletion. Western blot quantification is shown on the right (means \pm SEM, $n=3$ ). Sec61 is used as a loading control. 


\section{References}

1 López-Otín, C. and Overall, C. M. (2002) Protease degradomics: a new challenge for proteomics. Nat Rev Mol Cell Biol 3, 509-519.

2 Kleifeld, O., Doucet, A., Prudova, A., auf dem Keller, U., Gioia, M., Kizhakkedathu, J. N. and Overall, C. M. (2011) Identifying and quantifying proteolytic events and the natural $\mathrm{N}$ terminome by terminal amine isotopic labeling of substrates. Nat Protoc 6, 1578-1611.

3 Lemberg, M. K. (2011) Intramembrane proteolysis in regulated protein trafficking. Traffic, Blackwell Publishing Ltd 12, 1109-1118.

4 Yamano, K. and Youle, R. J. (2013) PINK1 is degraded through the N-end rule pathway. Autophagy.

5 Avci, D. and Lemberg, M. K. (2015) Clipping or Extracting: Two Ways to Membrane Protein Degradation. Trends Cell Biol. 25, 611-622.

6 Hampton, R. Y. and Sommer, T. (2012) Finding the will and the way of ERAD substrate retrotranslocation. Curr. Opin. Cell Biol. 24, 460-466.

7 Carlson, E. J., Pitonzo, D. and Skach, W. R. (2006) p97 functions as an auxiliary factor to facilitate TM domain extraction during CFTR ER-associated degradation. The EMBO Journal 25, 4557-4566.

8 Avci, D., Fuchs, S., Schrul, B., Fukumori, A., Breker, M., Frumkin, I., Chen, C.-Y., Biniossek, M. L., Kremmer, E., Schilling, O., et al. (2014) The Yeast ERIntramembrane Protease Ypf1 Refines Nutrient Sensing by Regulating Transporter Abundance. Molecular cell, Elsevier Inc. 56, 630-640.

9 Dean, R. A., Butler, G. S., Hamma-Kourbali, Y., Delbé, J., Brigstock, D. R., Courty, J. and Overall, C. M. (2007) Identification of candidate angiogenic inhibitors processed by matrix metalloproteinase 2 (MMP-2) in cell-based proteomic screens: disruption of vascular endothelial growth factor (VEGF)/heparin affin regulatory peptide (pleiotrophin) and VEGF/Connective tissue growth factor angiogenic inhibitory complexes by MMP-2 proteolysis. Mol. Cell. Biol., American Society for Microbiology 27, 8454-8465.

10 Dix, M. M., Simon, G. M. and Cravatt, B. F. (2008) Global mapping of the topography and magnitude of proteolytic events in apoptosis. Cell, Elsevier 134, 679-691.

11 Stoehr, G., Schaab, C., Graumann, J. and Mann, M. (2013) A SILAC-based approach identifies substrates of caspase-dependent cleavage upon TRAILinduced apoptosis. Molecular \& Cellular Proteomics, American Society for Biochemistry and Molecular Biology 12, 1436-1450.

12 Foresti, O., Rodriguez-Vaello, V., Funaya, C. and Carvalho, P. (2014) Quality control of inner nuclear membrane proteins by the Asi complex. Science 346, 751755.

13 Ong, S.-E., Blagoev, B., Kratchmarova, I., Kristensen, D. B., Steen, H., Pandey, A. and Mann, M. (2002) Stable isotope labeling by amino acids in cell culture, SILAC, as a simple and accurate approach to expression proteomics. Mol. Cell Proteomics 1, 376-386.

14 Pratt, J. M., Petty, J., Riba-Garcia, I., Robertson, D. H. L., Gaskell, S. J., Oliver, S. G. and Beynon, R. J. (2002) Dynamics of protein turnover, a missing dimension in proteomics. Mol. Cell Proteomics 1, 579-591. 
15 Aebersold, R. and Mann, M. (2003) Mass spectrometry-based proteomics. Nature 422, 198-207.

16 Sturm, M., Bertsch, A., Gröpl, C., Hildebrandt, A., Hussong, R., Lange, E., Pfeifer, N., Schulz-Trieglaff, O., Zerck, A., Reinert, K., et al. (2008) OpenMS - An opensource software framework for mass spectrometry. BMC Bioinformatics 9, 163.

17 Gietz, R. D. and Woods, R. A. (2002) Transformation of yeast by lithium acetate/single-stranded carrier DNA/polyethylene glycol method. Methods in Enzymology 350, 87-96.

18 Janke, C., Magiera, M. M., Rathfelder, N., Taxis, C., Reber, S., Maekawa, H., Moreno-Borchart, A., Doenges, G., Schwob, E., Schiebel, E., et al. (2004) A versatile toolbox for PCR-based tagging of yeast genes: new fluorescent proteins, more markers and promoter substitution cassettes. Yeast, John Wiley \& Sons, Ltd. 21, 947-962.

19 Kitada, S., Shimokata, K., Niidome, T., Ogishima, T. and Ito, A. (1995) A putative metal-binding site in the beta subunit of rat mitochondrial processing peptidase is essential for its catalytic activity. J. Biochem. 117, 1148-1150.

20 Frey, S., Pool, M. and Seedorf, M. (2001) Scp160p, an RNA-binding, polysomeassociated protein, localizes to the endoplasmic reticulum of Saccharomyces cerevisiae in a microtubule-dependent manner. J. Biol. Chem. 276, 15905-15912.

21 Tholen, S., Biniossek, M. L., Gessler, A.-L., Müller, S., Weisser, J., Kizhakkedathu, J. N., Reinheckel, T. and Schilling, O. (2011) Contribution of cathepsin L to secretome composition and cleavage pattern of mouse embryonic fibroblasts. Biol. Chem. 392, 961-971.

22 Rappsilber, J., Ishihama, Y. and Mann, M. (2003) Stop and go extraction tips for matrix-assisted laser desorption/ionization, nanoelectrospray, and LC/MS sample pretreatment in proteomics. Anal Chem 75, 663-670.

23 Shahinian, H., Loessner, D., Biniossek, M. L., Kizhakkedathu, J. N., Clements, J. A., Magdolen, V. and Schilling, O. (2014) Secretome and degradome profiling shows that Kallikrein-related peptidases 4, 5, 6, and 7 induce TGF $\beta-1$ signaling in ovarian cancer cells. Mol Oncol, Elsevier 8, 68-82.

24 Vizcaíno, J. A., Deutsch, E. W., Wang, R., Csordas, A., Reisinger, F., Ríos, D., Dianes, J. A., Sun, Z., Farrah, T., Bandeira, N., et al. (2014) ProteomeXchange provides globally coordinated proteomics data submission and dissemination. Nat Biotechnol 32, 223-226.

25 Deutsch, E. W. (2010) Mass spectrometer output file format mzML. Methods Mol. Biol., Humana Press, Totowa, NJ 604, 319-331.

26 Kessner, D., Chambers, M., Burke, R., Agus, D. and Mallick, P. (2008) ProteoWizard: open source software for rapid proteomics tools development. Bioinformatics 24, 2534-2536.

27 Kim, S. and Pevzner, P. A. (2014) MS-GF+ makes progress towards a universal database search tool for proteomics. Nat Comms 5, 5277.

28 Serang, O., MacCoss, M. J. and Noble, W. S. (2010) Efficient marginalization to compute protein posterior probabilities from shotgun mass spectrometry data. $\mathrm{J}$ Proteome Res 9, 5346-5357.

29 Serang, O. and Noble, W. S. (2012) Faster mass spectrometry-based protein inference: junction trees are more efficient than sampling and marginalization by 
enumeration. IEEE/ACM transactions on computational biology and bioinformatics / IEEE, ACM, IEEE 9, 809-817.

30 Nilse, L., Sigloch, F. C., Biniossek, M. L. and Schilling, O. (2015) Toward improved peptide feature detection in quantitative proteomics using stable isotope labeling. Prot. Clin. Appl. 9, 706-714.

31 Videm, P., Gunasekaran, D., der, B. S., Mayer, B., Biniossek, M. L. and Schilling, O. (2014) Automated peptide mapping and protein- topographical annotation of proteomics data $15,1-7$.

32 UniProt Consortium. (2015) UniProt: a hub for protein information. Nucleic Acids Res, Oxford University Press 43, D204-12.

33 Craig, R. and Beavis, R. C. (2004) TANDEM: matching proteins with tandem mass spectra. Bioinformatics, Oxford University Press 20, 1466-1467.

34 Keller, A., Nesvizhskii, A. I., Kolker, E. and Aebersold, R. (2002) Empirical statistical model to estimate the accuracy of peptide identifications made by MS/MS and database search. Anal Chem 74, 5383-5392.

35 Nesvizhskii, A. I., Keller, A., Kolker, E. and Aebersold, R. (2003) A statistical model for identifying proteins by tandem mass spectrometry. Anal Chem 75, 4646-4658.

36 Deutsch, E. W., Mendoza, L., Shteynberg, D., Slagel, J., Sun, Z. and Moritz, R. L. (2015) Trans-Proteomic Pipeline, a standardized data processing pipeline for largescale reproducible proteomics informatics. Prot. Clin. Appl. (Cutler, P., and Voshol, H., eds.) 9, 745-754.

37 Serang, O., Moruz, L., Hoopmann, M. R. and Käll, L. (2012) Recognizing uncertainty increases robustness and reproducibility of mass spectrometry-based protein inferences. J Proteome Res 11, 5586-5591.

38 Reiter, L., Claassen, M., Schrimpf, S. P., Jovanovic, M., Schmidt, A., Buhmann, J. M., Hengartner, M. O. and Aebersold, R. (2009) Protein identification false discovery rates for very large proteomics data sets generated by tandem mass spectrometry. Molecular \& Cellular Proteomics, American Society for Biochemistry and Molecular Biology 8, 2405-2417.

39 Li, Y. F., Arnold, R. J., Li, Y., Radivojac, P., Sheng, Q. and Tang, H. (2009) A bayesian approach to protein inference problem in shotgun proteomics. J. Comput. Biol. 16, 1183-1193.

40 Li, Y. F., Arnold, R. J., Radivojac, P. and Tang, H. (2012) Protein identification problem from a Bayesian point of view. Stat Interface 5, 21-37.

41 Han, D. K., Eng, J., Zhou, H. and Aebersold, R. (2001) Quantitative profiling of differentiation-induced microsomal proteins using isotope-coded affinity tags and mass spectrometry. Nat Biotechnol 19, 946-951.

42 Nilse, L. (2016) OpenMS: a modular, open-source workflow system for the analysis of quantitative proteomics data. In Proteome Informatics (Bessant, C., ed.) in press.

43 Sturm, M. and Kohlbacher, O. (2009) TOPPView: an open-source viewer for mass spectrometry data. J Proteome Res 8, 3760-3763.

44 Griss, J., Jones, A. R., Sachsenberg, T., Walzer, M., Gatto, L., Hartler, J., Thallinger, G. G., Salek, R. M., Steinbeck, C., Neuhauser, N., et al. (2014) The mzTab Data Exchange Format: Communicating Mass-spectrometry-based Proteomics and Metabolomics Experimental Results to a Wider Audience. Molecular \& Cellular Proteomics 13, 2765-2775. 
45 Huh, W.-K., Falvo, J. V., Gerke, L. C., Carroll, A. S., Howson, R. W., Weissman, J. S. and O'Shea, E. K. (2003) Global analysis of protein localization in budding yeast. Nature 425, 686-691. 